Voix et Images

volxetimages

\title{
De révolutions en circonvolutions. Répétition du récit et temps de l'histoire dans Prochain épisode
}

\section{Jean-François Hamel}

Volume 25, numéro 3 (75), printemps 2000

Normand Chaurette

URI : https://id.erudit.org/iderudit/201503ar

DOI : https://doi.org/10.7202/201503ar

Aller au sommaire du numéro

\section{Éditeur(s)}

Université du Québec à Montréal

\section{ISSN}

0318-9201 (imprimé)

1705-933X (numérique)

Découvrir la revue

\section{Citer cet article}

Hamel, J.-F. (2000). De révolutions en circonvolutions. Répétition du récit et temps de l'histoire dans Prochain épisode. Voix et Images, 25(3), 541-562. https://doi.org/10.7202/201503ar

\section{Résumé de l'article}

Si Prochain épisode présente une certaine muséification de l'histoire, souvent lue comme le signe d'un échec de la révolution, le roman n'en est pas moins porteur d'un questionnement sur les rapports entre, d'une part, la mémoire collective et les représentations de l'histoire et, d'autre part, l'expérience du présent historique. L'analyse des nombreux phénomènes de répétition narrative qui innervent le roman, ainsi que de certains propos d'Aquin sur l'Ulysse de Joyce, met en lumière les conceptions aquiniennes de la révolution, de la répétition et, plus généralement, du temps de l'histoire. 


\title{
De révolutions en circonvolutions. Répétition du récit et temps de l'histoire dans Prochain épisode
}

\author{
Jean-François Hamel, Université de Montréal / \\ Sorbonne nouvelle
}

\begin{abstract}
Si Prochain épisode présente une certaine muséification de l'bistoire, souvent lue comme le signe d'un échec de la révolution, le roman n'en est pas moins porteur d'un questionnement sur les rapports entre, d'une part, la mémoire collective et les représentations de l'bistoire et, d'autre part, l'expérience du présent bistorique. L'analyse des nombreux phénomènes de répétition narrative qui innervent le roman, ainsi que de certains propos d'Aquin sur lUlysse de Joyce, met en lumière les conceptions aquiniennes de la révolution, de la répétition et, plus généralement, du temps de l'bistoire.
\end{abstract}

N'allons pas croire que l'histoire est une grande pièce symphonique qui se développe avec thèmes, rappels et polyphonie de continu. L'histoire, qui englobe Hegel et Bergson dans la même glaise obscurante, ressemble beaucoup plus à un combat armé, combat interminable puisque, dans la brume épaisse, surgit à intervalles irréguliers un bataillon nouveau qui charge, sabre au clair, contre le flanc mou et blême du continu.

Hubert Aquin, "Calcul différentiel de la contre-révolution"

Le grand Giambattista Vico aurait-il, dans ses tiroirs morbides, quelques petites inventions névrogènes, quelques cercles vicieux dont il n'aurait pas eu le temps, de son vivant, de se servir? C'est un domaine à prospecter assurément.

Hubert Aquin, Point de fuite 


\section{Le bric-à-brac de l'histoire}

$\mathrm{Au}$ contraire de celle de Proust, l'œuvre romanesque d'Hubert Aquin n'est pas une recherche du temps perdu, mais bien une recherche du temps présent, d'une certaine présence du temps ${ }^{1}$. "Le temps me dévore, mais de sa bouche je tire mes histoires ${ }^{2}$, écrivait-il. De Prochain épisode, qui marque une première étape de cette recherche, celle du temps collectif, de sa mémoire et de l'histoire, jusqu'à Neige noire, qui exacerbe le rôle des mesures du temps dans une réflexion sur les temporalités phénoménologique et théologique, Aquin ne cesse de questionner les effets du savoir temporel sur l'expérience, les conséquences des multiples modes de représentation du temps sur la perception de sa présence et la possibilité de l'action. Une telle recherche présuppose que l'expérience temporelle, pour ainsi dire, ne va plus de soi.

À cet égard, Aquin se situe dans une lignée constituée autant de philosophes que d'écrivains, qui élaborent une critique de plus en plus sévère de l'histoire, interrogeant radicalement la possibilité de son expérience dans le cadre de la modernité. Une image insiste, ne cesse de revenir, jusqu'à trouver dans Prochain épisode une énième occurrence: l'histoire comme foire bariolée et vertigineuse, vaste musée aux murs surchargés par les restes des siècles passés, capharnaüm sidérant jusqu'au spectateur le plus sceptique. Face à cet amas de vestiges, lourds et accaparants, l'effet est toujours le même pour celui qui y porte son regard: la statufication qui le constitue subitement en pièce de ce musée tout-puissant lui dénie toute possibilité d'action au sein du temps historique. C'est en quelque sorte la femme de Loth regardant derrière elle: la transcendance qui transforme en statue de sel n'est plus Dieu ou Sodome en flammes, c'est l'histoire elle-même. Que la possibilité d'une expérience véritable du temps historique se soit raréfiée depuis deux siècles, c'est ce que paraît véhiculer cette image faisant retour.

Walter Benjamin en découvre une première occurrence dans La peau de chagrin de Balzac et l'interprète comme "l'élément pathologique dans la représentation de la civilisation ${ }^{3}$ : : Raphaël, le héros du roman, s'aventure chez un marchand de curiosités où la totalité de l'histoire semble se donner en représentation:

Au premier coup d'œil, les magasins lui offrirent un tableau confus, dans lequel toutes les ouvres humaines et divines se heurtaient. [...] Le commencement du monde et les événements d'hier se mariaient avec une

1. Cet article s'inscrit dans un projet de recherche doctoral intitulé : La narrativité à l'épreuve de l'bistoire. Poétique et politique des récits de la répétition dans la modernité littéraire.

2. Hubert Aquin, Neige noire, Montréal, Bibliothèque québécoise, 1997 [1974], p. 277.

3. Walter Benjamin, Paris, capitale du $x x^{e}$ siècle. Le livre des passages, trad. J. Lacoste, Paris, Cerf, 1989, p. 506. 
grotesque bonhomie. [...] Tous les pays de la terre semblaient avoir apporté là quelques débris de leurs sciences, quelques échantillons de leurs arts. C'était une espèce de fumier philosophique auquel rien ne manquait, ni le calumet du sauvage, ni la pantoufle vert et or du sérail, ni le yatagan du Maure, ni l'idole des Tartares. [...] La vue de tant d'existences nationales ou individuelles, attestées par ces gages humains qui leur survivaient, acheva d'engourdir les sens du jeune homme ${ }^{4}$.

Dans ce labyrinthique espace où les différentes strates du devenir se donnent simultanément sur un même plan, l'expérience historique du jeune homme s'amenuise comme une peau de chagrin parce que, selon Benjamin, "les richesses qui se trouvent ainsi collectionnées dans l'aérium de la civilisation apparaissent désormais comme identifiées pour toujours ${ }^{5}$. La représentation chosiste de l'histoire, comme bazar ou magasin de curiosités, exprime, selon Benjamin, un déclin de l'expérience du présent de l'histoire - explicable tant par un arrêt du devenir provoqué par la rupture de la tradition (qui assurait une hiérarchie dans la mémoire et un cadre collectif à l'expérience) que par l'intensification de la production de masse qui transforme en objet de consommation courante jusqu'à l'événementialité. C'est encore cette dispersion de la mémoire et cette réification de l'histoire que Baudelaire aurait exprimées dans ces vers écrits deux décennies après le roman de Balzac: "J'ai plus de souvenirs que si j'avais mille ans. [...] Je suis un boudoir plein de roses fanées, où git tout un fouillis de modes surannées ${ }^{6}$. "Le sujet de l'histoire se perd donc dans son objet, jusqu'à le devenir, jusqu'à se réifier à son tour, le temps semblant s'immobiliser sous le poids de pures remémorations, de réminiscences abolissant la perception de son passage. L'histoire apparaît dès lors comme un revenant sans voix; elle fait retour, mais sans pour autant ramener avec elle le sens de son événementialité. Nietzsche file lui aussi la métaphore en définissant, parmi les formes d'histoire qui peuvent, malgré toutes ses réserves, ne pas nuire à la vie, l'histoire traditionaliste comme poursuite du "bric-à-brac ancestral ${ }^{7}$, Le danger de cette "aveugle fureur de collection", de cette "passion universelle pour tout ce qui est ancien", est que trop souvent "elle ne sait que conserver l'histoire, non pas l'engendrer ${ }^{8}$ : elle n'a donc que le pouvoir de reproduire les événements passés, jamais de produire l'imprévisible nouveauté du devenir ou même la singularité d'une événementialité antérieure. Perdu dans un passé privé de son avenir, le sujet stérilise son héritage historique en le

\footnotetext{
4. Honoré de Balzac, La peau de chagrin, Paris, Garnier/Flammarion, 1971 [1831], p. 71.

5. Walter Benjamin, op. cit., p. 46.

6. Charles Baudelaire, "Spleen", Les fleurs du mal, Paris, Le livre de poche, 1968 [1859], p. 86.

7. Friedrich Nietzsche, "De l'utilité et des inconvénients de l'histoire pour la vie", Considérations inactuelles I et II, trad. P. Rusch, Paris, Gallimard, coll. "Folio essais", 1995 [1874], p. 109.

8. Ibid., p. 112.
} 
muséifiant. Il croit écrire sa propre histoire par le classement méticuleux du legs des générations passées.

Dans Prochain épisode ${ }^{9}$, Hubert Aquin livre à son tour au moins deux grandes scènes muséales où le protagoniste du roman se laisse hypnotiser par la grandeur du passé, où son désir d'un instant révolutionnaire se raréfie peu à peu, jusqu'à se dissiper complètement dans la contemplation de l'immobilité itérative de l'histoire. La première scène a lieu au château d'Échandens où, attendant le retour de son ennemi (le contrerévolutionnaire $\mathrm{H}$. de Heutz), le narrateur sombre dans une léthargie funeste au cœúr de "cette retraite empreinte de douceur où s'exprime un vouloir-vivre antique" $(P E, 122)$. Dans le "musée obscur" $(P E, 132)$ qu'est ce château chargé de souvenirs, "drapé dans ses époques et ses styles" ( $P E$, 122), il se laisse emporter par les eaux stériles d'une histoire dans laquelle toute révolution a perdu sa violence, où les flammes insurrectionnelles se sont définitivement laissées submerger par le cours du temps, où le "vouloir-vivre" n'exprime plus qu'un désir récessif, une passion sans ancrage dans le présent. La seconde scène, elle aussi annonciatrice d'un échec, se produit chez l'antiquaire montréalais Menndellsohn de la rue Craig, rue nommée en l'honneur du gouverneur anglais du Bas-Canada qui réprima sévèrement les rébellions de 1837-1838. Décrivant les vieilleries et les raretés qui meublent la boutique, Aquin prépare le lecteur à l'échec final du protagoniste en réinscrivant une dernière fois son projet révolutionnaire dans le bric-à-brac ancestral de l'histoire où tout événement semble s'empoussiérer comme sur la page d'un livre écrit depuis toujours.

Et comme j'avais une heure à tuer, je suis descendu, par la rue SaintFrançois-Xavier, jusqu'à la rue Craig, et je suis entré chez Menndellsohn. J'adore cet endroit; quand j'y pénètre, j'ai toujours le pressentiment que je vais découvrir la montre de poche du général Colborne ou le revolver avec lequel Papineau aurait mieux fait de se suicider. $(P E, 155)$

En sortant du capharnaüm, le narrateur fait quelques pas dans le VieuxMontréal, entre les murs historiques de la cité, se rend jusqu'à l'église Notre-Dame où deux policiers attendent son arrivée. Fait prisonnier dans les restes obsédants de l'histoire de ses ancêtres, le narrateur n'a plus qu'à projeter l'écriture d'un roman, celui du prochain épisode évoqué dans les dernières pages. Le narrateur se trouve ainsi doublement emmuré: d'abord par l'histoire qui lui dénie toute influence sur son propre devenir, ensuite par son récit qui le ramène à la case départ dans la répétition d'un projet romanesque interminable.

L'histoire apparaît donc dans Prochain épisode comme une substance narcotique, un nouvel opium du peuple qui engourdit chacun de ses

9. Nous utilisons l'édition critique du roman à laquelle renvoient toutes les indications de folio précédées du sigle $P E$ : Hubert Aquin, Procbain épisode, Montréal, Bibliothèque québécoise, 1998 [1965]. 
membres ${ }^{10}$. Sa pesanteur se manifeste aussi dans les essais d'Aquin (sous la forme d'une "identité atavique"), par une "fatigue culturelle ${ }^{11}$ " généralisée, engageant l'écrivain dans une esthétique du deuil, dans un répétitif "art de la défaite ${ }^{12}$ ". Cette "catatonie nationale" $(P E, 54)$ pourrait certes s'expliquer par l'inscription à la fois diachronique et synchronique de Prochain épisode dans l'histoire du Québec. Que ce soit par l'ambiguité constitutive des origines de la société québécoise - qui oscille toujours entre la ferveur messianique et la désillusion, entre l'utopie de l'Europe et le rêve d'une Amérique française effacé par la Conquête - , ou encore par le long travail de constitution d'une littérature et d'une historiographie nationales qui, en voulant réfuter les opinions de Durham, leur accorde plus d'importance que le pauvre Anglais n'aurait pu le croire, l'histoire de la société et de littérature québécoises semble s'élaborer comme une suite incessante de réponses, toujours provisoires, à la question du sens de l'Origine ${ }^{13}$. Si Prochain épisode est tributaire de ce questionnement sur les commencements, il l'est aussi de la métamorphose qui s'est opérée pendant les années soixante: une volonté d'émancipation, héritée du projet de la modernité, selon lequel l'identité d'une collectivité repose non sur son origine, mais sur sa finalité. Or, que la question du sens de l'histoire se pose depuis le passé, selon le mythe de l'Origine, ou face à l'avenir, par la vertu d'une Fin qui le chargera de signification, c'est toujours le présent historique qui est dévalué, inscrit dans une série dont il n'est qu'à peine un point de passage, jamais le levier. L'histoire n'est plus alors qu'un vaste présentoir où, à côté des vieilleries et des curiosités d'un passé jamais véritablement révolu, l'avenir même est classé comme déjà advenu, puisqu'il devra nécessairement advenir. D'où ce bric-à-brac de l'histoire, cette muséification du devenir, qui sont chez Aquin tant l'expression d'une Origine sans cesse reprise, parce que née de l'échec et de la défaite, que celle d'une Fin non moins repoussée, toujours à venir parce que asymptotique.

Toutefois, comme si Prochain épisode n'était qu'un reflet de ces discours, la critique soutient trop souvent que le roman est l'expression de ces échecs qui se répètent dans l'histoire. En d'autres mots, Aquin aurait capitulé devant cette muséification par laquelle il décrivait la perte

10. Rappelant la devise du Québec, "Je me souviens", le narrateur note: "Je m'emplis de père en fils d'anticorps; je me saoule, fidèle à notre amère devise, d'une boisson nitrique qui fait de moi un drogué." $(P E, 31)$

11. Hubert Aquin, "La fatigue culturelle du Canada français ", Mélanges littéraires II. Comprendre dangereusement, Montréal, Bibliothèque québécoise, 1995, p. 102.

12. Id., "L'art de la défaite. Considérations stylistiques ", Mélanges littéraires II. Comprendre dangereusement, p. 131-144.

13. Fernand Dumont a montré l'importance de cette question dans Genèse de la sociêté québécoise, Montréal, Boréal, coll. "Compact ", 1996 [1993]. Sur le travail de deuil et de symbolisation de l'Origine chez Aquin, voir Jacques Cardinal, Le roman de l'bistoire. Politique et transmission du nom dans Prochain épisode et Trou de mémoire de Hubert Aquin, Montréal, Balzac, 1993. 
de son héros ${ }^{14}$. Prochain épisode serait donc le roman de la révolution, mais plus encore de son échec. Or, le roman peut être lu selon la perspective inverse, non comme une soumission, consciente ou non, au poids de l'histoire et à sa fausse clarté, mais bien plutôt comme une critique sévère, sous une forme compulsivement parodique, assénée à coups de répétitions, de son obscur refoulé. Le malentendu naît de ce que l'inscription du roman dans son contexte d'énonciation et dans l'histoire tant littéraire que politique, qui constitue une des voies fondamentales d'interprétation, élude la part d'interrogation propre que comporte sa poétique à l'égard de l'histoire. Car non seulement Prochain épisode s'enracine dans un horizon historique clairement défini, comme toute œuvre d'art, mais il s'en échappe en posant l'histoire - entendue comme totalité du devenir - comme son enjeu premier. En effet, le roman est une scène où s'affrontent différentes représentations historiques, où s'ouvre une polémique entre des chronographies hétérogènes, entre différents modes d'écriture du temps. Ne succombant en rien au bric-à-brac de l'histoire, Prochain épisode en dresse au contraire la caricature et s'en fait un masque: un "masque de grand déprimé" $(P E, 58)$ qui parodie ce lieu commun selon lequel il faudrait, servilement, suivre le cours de l'histoire. L'inachèvement du roman, sa fragmentation, l'échec même de son protagoniste, mais surtout les phénomènes internes et externes de répétition qui l'innervent, constituent précisément la réussite critique de Prochain épisode à l'égard d'une certaine impasse historique, l'issue permettant de réaffirmer l'importance de penser l'histoire au présent.

14. À cet égard, l'interprétation de Józef Kwaterko - malgré toute la rigueur avec laquelle celui-ci révèle la maîtrise de la mise en discours que réalise le roman à partir des idéologies plurielles et hétérogènes traversant la Révolution tranquille - demeure symptomatique de certaines idées reçues de la critique aquinienne. "Hallucinée et fantasmée, l'histoire québécoise, idéologème central du roman, ne peut être représentée que par une pluralité de discours-greffons qui sans cesse contrecarrent l'accomplissement romanesque au lieu de le favoriser. Ceci nous amène à conclure que la polyphonie romanesque opère ici une ambiguïté idéologique qui coupe les amarres référentielles du texte et demeure reliée au discours propre du narrateur. Ce discours nous apparaît comme irréductible à la dialectisation, mais bien plus comme résultat d'un conflit idéologique et textuel non dépassé: celui du dialogue d'une voix posée comme réelle et interprétative avec des voix fictives qu'elle exhibe, ironise ou parodie, mais qu'elle n'arrive nullement à briser ni à réduire au silence. Dans cette perspective, la polyphonie dans Prochain épisode signifie bien l'orchestration des voix qui fusionnent en une seule conscience (une conscience déchirée) mais qui ne permettent pas de construire une représentation du monde cohérente. " (Józef Kwaterko, Le roman québécois de 1960 à 1975. Idéologie et représentation littéraire, Longueuil, Le Préambule, coll. "L'univers des discours ", 1989, p. 154.) Malgré la subtilité de l'analyse qui précède cette conclusion, l'affirmation d'une absence de représentation cohérente du monde reconduit ici selon une approche sociocritique le sempiternel constat d'échec que la majorité des critiques, de tous horizons, attribuent au roman depuis plus de trente ans. Néanmoins, d'autres interprétations ont tempéré ce constat, entre autres celle, aussi pénétrante que brève, de Gilles Marcotte dans "Le romancier comme cartographe ", Le roman à l'imparfait, Montréal, Typo, 1989 [1976], p. 227-252. 


\title{
Le temps héraclitéen
}

Le roman s'ouvre sur un lyrisme qui, en quelques phrases seulement, met en place la problématique narrative qui le traversera de part en part.

\begin{abstract}
Cuba coule en flammes au milieu du lac Léman pendant que je descends au fond des choses. Encaissé dans mes phrases, je glisse, fantôme, dans les eaux névrosées du fleuve et je découvre, dans ma dérive, le dessous des surfaces et l'image renversée des Alpes. Entre l'anniversaire de la révolution cubaine et la date de mon procès, j'ai le temps de divaguer en paix, de déplier avec minutie mon livre inédit et d'étaler sur ce papier les mots clés qui ne me libéreront pas. J'écris sur une table à jeu, près d'une fenêtre qui me découvre un parc cintré par une grille coupante qui marque la frontière entre l'imprévisible et l'enfermé. Je ne sortirai pas d'ici avant échéance. Cela est écrit en plusieurs copies conformes et décrété selon des lois valides et par un magistrat royal irréfutable. Nulle distraction ne peut donc se substituer à l'horlogerie de mon obsession, ni me faire dévier de mon parcours écrit. $(P E, 5)$
\end{abstract}

L'ouverture du roman manifeste avec une insistance singulière la question de la temporalité : on y évoque tour à tour une échéance, un anniversaire, une révolution, la date d'un procès, l'borlogerie d'une obsession, obsession qui apparaîtra de plus en plus clairement comme celle du temps et, plus précisément encore, de l'histoire. Mais c'est par deux métaphores conflictuelles que se présente de la façon la plus aiguë la problématique du roman: tout au long de Prochain épisode, l'eau et le feu ne cesseront de revenir hanter le récit, qui réactualisera ainsi certains éléments de la philosophie d'Héraclite qu'Aquin ne pouvait que connaître, au moins pour avoir été un si grand lecteur de Nietzsche.

La cosmologie héraclitéenne expliquait l'unité de toute chose, malgré la puissante dialectique des oppositions, par une seule et unique substance primordiale: le feu, puissance qui s'échange contre toute chose et rend possible tant la création que la destruction dans un mouvement cyclique. Le philosophe d'Éphèse soutenait aussi une doctrine de l'être en continuel mouvement, en perpétuel devenir, représentée par la métaphore, devenue lieu commun, du mouvement des eaux: "Tu ne peux pas descendre deux fois dans un même fleuve; car de nouvelles eaux coulent toujours sur toi ${ }^{15}$. " De même, le feu et les flammes cubaines sont pour le narrateur révolutionnaire le moteur de l'histoire, l'élément primordial à l'origine comme à la fin d'une période ou d'un régime politique. Et l'eau, associée ici au lac Léman mais renvoyant aussi à d'autres référents au fil du texte ${ }^{16}$, correspond au processus historique, au passage du temps, le

15. Voir Les penseurs grecs avant Socrate. De Thalès de Milet à Prodicos, Paris, Garnier/ Flammarion, 1964, p. 71-85; Émile Brébier, Histoire de la philosophie I. Antiquité et Moyen Âge, Paris, Presses universitaires de France, coll. "Quadrige ", 1985, p. 48-53.

16. Une analyse de la prégnance du thème de l'eau dans les romans d'Aquin révélerait probablement que le temps constitue un véritable objet de désir, comme l'indique la fréquence des rapprochements entre la femme aimée et le paysage aquatique. Il y a bel 
plus souvent douloureux pour le narrateur enfermé, mais essentiel pour son protagoniste. L'eau symbolise aussi, lorsqu'elle est contenue entre les rives d'un lac, l'immobilité apparente de l'histoire. L'opposition entre le feu et l'eau se pose ainsi comme "frontière entre l'imprévisible et l'enfermé", entre l'insurrection et la tradition, entre le bris et la continuité.

C'est l'antagonisme de ces deux temporalités, celle de la rupture brûlante et enflammée de la révolution et celle du cours mesuré de l'histoire, comme passage et devenir mais aussi comme poids et lenteur, qui constitue le vecteur du récit. Du seul point de vue épistémologique, le questionnement essentiel du roman, relevant aussi de cette tension dialectique, concerne la possibilité d'inscrire la révolution dans l'histoire, de faire le récit de la révolution alors que celle-ci se veut précisément la fracture d'un récit antérieur. La difficulté d'une telle inscription passe par l'image de Cuba coulant en flammes au fond du lac Léman, le feu risquant toujours de s'éteindre dans les eaux calmes de l'histoire.

\section{La géométrie du cercle}

Outre cette métaphore héraclitéenne, Aquin imprègne son roman d'un motif géométrique, le cercle et ses dérivés (spirales, courbes, boucles), qui renvoient à la figure d'un destin insurmontable, à un échec infiniment répété. Dans un cercle vicieux qui fait du passé son seul avenir, le narrateur évoque tour à tour sa "chute spiralée" $(P E, 44)$, son "écriture courbée" $(P E, 115)$, ou "l'énigme dans laquelle [il s']enroule" $(P E, 140)$. Autant de mouvements circulaires dont le point d'arrivée coïncide avec l'origine: "Toutes les courbes que j'enlace passionnément et les vallées que j'escorte me conduisent implacablement dans cet enclos peuplé de fantômes." (PE, 143) Ou encore: "[J]e suis cet homme anéanti qui tourne en rond sur les rivages du lac Léman. " $(P E, 160)$ Les pages (et les jours) du narrateur se soumettent à la même ritournelle, inlassablement reprise. C'est que la lutte entre la révolution et l'histoire se joue d'emblée dans Prochain épisode sur.le terrain de la répétition.

"Je dérape dans les lacets du souvenir. " $(P E, 41)$ En effet, la répétition surgit en premier lieu dans l'autonarration lorsque le narrateur raconte son enfermement et sa désespérance à la suite de son arrestation pour activités révolutionnaires ${ }^{17}$. Il évoque des moments passés avec $\mathrm{K}$, la

et bien une femme-pays dans Prochain épisode qui en appelle à un territoire mythique toujours à venir, comme dans la poésie de Miron ou de Chamberland, mais il existe aussi une femme-temps qui renvoie au désir d'une expérience authentique et immédiate du devenir. Pour une recension et une analyse de certaines occurrences, voir M.E. Kidd, "La thématique de l'eau dans l'œuvre d'Hubert Aquin ", Voix et Images, vol. IV, $\mathrm{n}^{\circ} 2$, décembre 1978, p. 264-271.

17. La situation d'énonciation du narrateur est évidemment très proche de celle d'Aquin au moment d'écrire le roman. Il faut rappeler que le 18 juin 1964 Aquin annonce par un communiqué de presse qu'il quitte son poste de vice-président régional du Rassemble- 
femme aimée: d'abord une promenade où se mêlent des images des rébellions de 1837-1838 au Bas-Canada; puis une nuit d'amour à l'Hôtel d'Angleterre où Lord Byron se serait arrêté avant de participer aux insurrections grecques contre la domination turque au début du $\mathrm{XIX}^{\mathrm{e}}$ siècle; enfin, une autre nuit d'amour, un 24 juin, fête nationale des Québécois, dans laquelle révolution et orgasme se joignent dans une même urgence insurrectionnelle. Les souvenirs, constamment ressassés, presque fétichisés, se lient tous étroitement à des anecdotes révolutionnaires qui brisent (comme la révolution le fait avec l'histoire) le fil du récit d'espionnage. Une première forme de répétition apparaît ici par ressouvenirs ou anamnèses, qui eux-mêmes reprennent les obsessions révolutionnaires du récit: répétition et révolution cö̈ncident donc par la logique mémorielle de la narration. Or, le ressouvenir n'est pas ici que la répétition par la mémoire d'un événement personnel, mais la répétition même des moments où l'histoire risqua de se briser ou se brisa effectivement au profit d'une autre histoire. Par la médiation de la mémoire, l'autonarration présente donc la répétition d'événements révolutionnaires, de moments historiques pour lesquels, lors de leur avènement, n'existait apparemment aucun précédent. Ce retour de ce qui est sans passé, d'une parfaite nouveauté, joue donc - à l'image de tout le roman - sur les deux acceptions du terme révolution: à la fois cycle, retour, comme à propos des astres, et rupture, fracture, renversement d'un ordre établi. Ce qui revient hanter la mémoire du narrateur, c'est ce qui n'a pas de mémoire et qui pourtant ne cesse de faire retour.

La métanarration n'est pas moins préoccupée par la répétition. Donnant à voir les difficultés de la poétique de l'œuvre en chantier et la profonde ironie qu'il y a à élaborer un roman d'espionnage fait de poursuites et de dénouements abracadabrants depuis une situation d'emprisonnement, la métanarration est issue d'un regard transcendant sur le roman. La première est liée à l'intertextualité générique, inscrivant le roman dans un genre préexistant. "J'éprouve une grande sécurité à me pelotonner mollement dans le creuset d'un genre littéraire aussi bien défini. Sans plus tarder, je décide donc d'insérer le roman qui vient dans le sens majeur de la tradition du roman d'espionnage." $(P E, 6)$ Mais cette première résolution consistant à perpétuer - sinon répéter - une certaine tradition narrative ne sera jamais vraiment respectée et le paradoxe

ment pour l'indépendance nationale (RIN) pour plonger dans la lutte révolutionnaire et la clandestinité. Le 5 juillet suivant, il sera arrêté pour port d'arme au cours d'une tentative manquée de vol de voiture. Le 15 juillet, le lendemain de son procès, Aquin est transféré de la prison commune de Montréal à l'Institut psychiatrique Albert-Prévost où il commence la rédaction de Prochain épisode. Le roman est ainsi la reprise romanesque d'une situation réelle, phénomène ne correspondant pas ici uniquement à une entreprise autobiographique, mais s'inscrivant aussi dans la longue série des phénomènes de reprise et répétition narrative qui structure l'œuvre. 
qu'elle contient ne cessera de resurgir. Car comment inscrire une révolution dans un schéma narratif que l'on reproduit mécaniquement? Comment représenter une rupture de l'histoire en reprenant un mode de narration aux profondes racines historiques?

Ce roman métissé n'est qu'une variante désordonnée d'autres livres écrits par des écrivains inconnus. Pris dans un lit de glaise, je suis le cours et ne l'invente pas. Ceci vaut pour tout ce que j'écris: me voici au fond d'une impasse où je cesse de vouloir avancer. [...] Mais alors si ce choc qui anéantit mon ambition d'originalité écrite me terrasse à ce point; $s i$ je suis subitement privé de ma raison d'écrire parce que je perçois mon livre à venir comme prédit et marqué d'avance, selon la cotation Dewey, d'un coefficient infime d'individuation et que, dans un même temps, je n'en cesse pas pour autant de vouloir écrire, c'est donc que l'écriture ne deviènt pas inutile du seul fait que je la départis de sa fonction d'originalité et que justement cette fonction génétique ne la résume pas. Du moins, l'ambition d'originalité n'est pas seule à valoriser l'entreprise littéraire. On peut donc entreprendre un roman d'espionnage dont l'action se déroule comme une anomalie sur les bords du lac Léman, avec un autre motif que d'en faire une œuvre unique. L'originalité est un idéal de preux: c'est le Graal esthétique qui fausse toute expédition. Jérusalem seconde, cette unicité surmultipliée n'est rien d'autre qu'une obsession de croisés. ( $P E, 88)$

Le refus de l'originalité littéraire par l'apparente acceptation des paradigmes romanesques préexistants révèle la nature répétitive de l'acte narratif, le caractère inévitable de l'intertextualité, consciente ou non, de toute entreprise d'écriture et l'annihilation du fantasme démiurgique en elle. Mais le narrateur aquinien laisse aussi entendre que se dévoile une obscure originalité propre au refus de ce "Graal esthétique", une singularité irréductible et secrète à même la répétition des sédiments de la tradition narrative. Mystérieusement, une première fois, il est suggéré que la répétition n'est pas uniquement répétition de l'identique, mais que ce mouvement de reprise produit sa propre altérité. À même le cercle se trouverait donc une issue.

Une deuxième transcendance imprègne encore les réflexions sur le roman en cours, celle de l'histoire entendue clairement comme une écriture : "La nuit historique semble sécréter l'encre de chine dans laquelle je distingue trop de formes fuyantes." $(P E, 16)$ La narration et la métanarration s'insèrent en effet malgré elles dans un métarécit complexe. Le poids de l'histoire apparaît très tôt au narrateur comme une volonté extérieure, presque divine, qui le contraint à la répétition d'une narration première écrite par autrui, le soumet à une intrigue qui le précéde, l'inscrit lui-même dans un récit antérieur:

Rien n'est libre ici, rien: même pas cette évasion fougueuse que je téléguide du bout des doigts et que je crois conduire quand elle m'efface. Rien! Pas même l'intrigue, ni l'ordre d'allumage de mes souvenirs, ni la mise au tombeau de mes nuits d'amour, ni le déhanchement galiléen de mes femmes. Quelque chose me dit qu'un modèle antérieur plonge mon improvisation 
dans une forme atavique et qu'une alluvion ancienne étreint le fleuve instantané qui m'échappe. Je n'écris pas, je suis écrit. Le roman incréé me dicte le mot à mot que je m'approprie, au fur et à mesure, selon la convention de Genève régissant la propriété littéraire. Je crée ce qui me devance et pose devant moi l'empreinte de mes pas imprévisibles. [...] Tout m'attend. Tout m'antécède avec une précision que je dévoile dans le mouvement même que je fais pour m'en approcher. J'ai beau courir, on dirait que mon passé antérieur a tracé mon cheminement et proféré les paroles que je crois inventer. $(P E, 85)$

Cet aveu d'impuissance du narrateur, qui se révèle par l'inscription du récit dans une trame qui le précède et le détermine dans son devenir même, voile malgré tout une certaine réussite, car le discours qui exprime la répétition à l'œuvre dans l'histoire échappe au récit. Le discours l'emportant dans ces passages sur le récit, le roman se trouve brisé dans sa narrativité même ainsi que dans sa participation au genre romanesque. Telle une incohérence qui viendrait souligner la contingence des conventions narratives régissant jusqu'au métarécit qu'est l'histoire, le discours apparaît comme l'élément révolutionnaire du roman, le moyen par lequel se trouve morcelée la structure narrative, perçue comme assujettissement puisqu'elle relève nécessairement de l'histoire. Comme si dire la répétition, c'était déjà s'en extraire.

Mais l'échec du récit face à la révolution, cette façon qu'ont encore les flammes cubaines de scintiller jusque dans les eaux du lac Léman, n'en dissipe pas moins le désir d'une autre histoire que la narration actancielle tentera en vain de prendre en charge. La révolution demeure, malgré la rupture qu'elle inflige à la narration, une dialectique, une agonique entre deux narrations concurrentes. La volonté révolutionnaire, bien qu'elle soit toujours repoussée dans une préhistoire, n'en cherche pas moins démesurément, comme par une compulsion de répétition, à passer à l'histoire.

\section{La ruse de la narrativité historique}

La narration actancielle est l'expression du désir sans cesse déçu d'une histoire cohérente. La structure du récit policier se présente comme une lutte des narrations médiatisée par la poursuite des deux antagonistes autour du lac Léman et, selon la symbolique qui se dégage du roman, autour de l'histoire: s'opposent le narrateur-révolutionnaire au service du Front de libération du Québec et le contre-révolutionnaire, H. de Heutz, probablement agent de la Gendarmerie royale du Canada et, par ailleurs, professeur d'histoire. Le contre-révolutionnaire apparaît en effet une première fois dans l'annonce d'une conférence: "Mardi le $1^{\text {er }}$ août, le professeur H. de Heutz de l'Université de Bâle, donnera une conférence sur "César et les Helvètes" sous les auspices de la Société d'histoire de la Suisse romande." $(P E, 9) \mathrm{H}$. de Heutz est donc l'homme qui non seulement représente l'histoire, mais plus significativement qui connaît 
l'inscription d'une première insurrection, celle des Helvètes, dans le cours historique des événements ${ }^{18}$.

Dans la chasse à l'homme qui traverse le roman, $\mathrm{H}$. de Heutz est le premier à s'emparer de son opposant, alors qu'il est suivi par ce dernier dans les ruelles du quartier Carouge, ancien refuge des révolutionnaires russes. À la suite de sa capture, le révolutionnaire est interrogé par son ennemi; désarmé et étourdi, le narrateur-protagoniste ne voit qu'une seule issue à sa position d'infériorité: "Il me fallait élaborer une riposteéclair et puisque je n'avais plus d'arme à dégainer, vider mon chargeur dialectique sur cet inconnu dressé entre le jour et moi." $(P E, 54)$ Le révolutionnaire pris au piège n'a plus qu'un recours: "improviser surle-champ un scénario passe-muraille" $(P E, 54)$, utiliser la narration comme feinte, user de la violence d'un récit pour ébranler la contenance de son ennemi, c'est-à-dire revêtir son "masque de grand déprimé" $(P E, 58)$. La stratégie réussit et permet au narrateur de faire à son tour $\mathrm{H}$. de Heutz prisonnier. Toutefois, au moment de son exécution dans le bois de Coppet, le captif entame une longue défense qui reprend littéralement le récit que le narrateur avait inventé de toutes pièces quelques heures plus tôt pour se défaire de son emprise. En quelques phrases, $\mathrm{H}$. de Heutz hypnotise le narrateur en lui resservant sa propre narration, celle d'un grand déprimé qui a laissé femme et enfants derrière lui pour leur éviter la honte de ses échecs.

J'ai le doigt sur la gâchette: je n'ai qu'à presser et j'exauce son vœu. Pourtant j'hésite encore. L'histoire qu'il persiste à me raconter me' pose une énigme. Pourquoi a-t-il choisi de me réciter exactement la même invraisemblance que je lui ai servie sans conviction ce matin même, alors qu'il me tenait en joue dans le grand salon d'Echandens? [...] Si j'en suis rendu à analyser les intentions profondes de son comportement avec moi, peut-être, au fond, suis-je sur le point de tomber dans le piège qu'il m'a tendu, et que je réagis exactement comme il l'a voulu? Ma fascination même - ainsi que son corollaire de doute méthodique et d'hésitation -, il l'a provoquée sciemment. [...] Tout se ralentit. Mes pulsations mêmes semblent s'espacer. L'agilité supersonique de mon esprit s'affaisse soudainement sous le charme maléfique de $\mathrm{H}$. de Heutz. Je m'immobilise, métamorphosé en statue de sel, et ne puis m'empêcher de me percevoir comme foudroyé. Un événement souverain est en train de se produire, en cet instant où j'occupe un espace réduit dans un bois charmeur qui surplombe Coppet. ( $P E, 81-83)$

Le narrateur fait ici l'expérience du pouvoir de la narration de l'Autre, et surtout de la dialectique avec laquelle s'intègre à son propre déroulement les éléments les plus audacieux de sa propre narration. Métaphorique-

18. On remarquera aussi, comme le souligne Jacques Allard dans son introduction à l'édition critique du roman, qu'il demeure une indécision autour du nom du contrerévolutionnaire - " $\mathrm{H}$. de Heute ou $\mathrm{H}$. de Heutz", hésite Aquin à deux reprises - et que boute signifie aujourd'bui en allemand, ce qui semble un indice supplémentaire de la problématique temporelle du roman. 
ment, c'est le geste révolutionnaire qui s'intègre au continu de l'histoire, le feu qui s'éteint sous le flux des eaux. S'immobilisant, tant au plan physique qu'au plan narratif, le révolutionnaire devient une statue de sel pour avoir porté son regard en arrière, pour s'être laissé charmer par une histoire dans laquelle il se trouve inscrit et dont il se croyait pourtant maître. La répétition, par $\mathrm{H}$. de Heutz, de la feinte narrative du narrateur, dévoile peu à peu la structure d'une autre répétition: c'est le révolutionnaire qui, malgré lui, répète le geste que l'histoire attend de lui, qui, par sa posture même, participe à l'immobilité itérative de l'histoire et de la contre-révolution. Le révolutionnaire, étourdi par la ruse narrative de H. de Heutz, le laisse s'enfuir, incapable de tuer celui qui apparaît de plus en plus clairement comme son double ${ }^{19}$.

Après avoir repris ses esprits, le narrateur décide de revenir à l'assaut et pénètre dans le château qu'habite $\mathrm{H}$. de Heutz pour l'y attendre et le tuer. Dans le château, il se surprend à apprécier cette "crypte lumineuse" $(P E, 123)$, puis, peu à peu, il s'identifie à l'ennemi dont il tente de reconnaître la figure entre un buffet Louis XIII, des livres anciens (dont l'Histoire de Jules César du général Stoffel), et un tableau de Benjamin West intitulé La mort du général Wolfe. "Revêtu des attributs ornementaires de $\mathrm{H}$. de Heutz, entouré par les meubles qu'il a lui-même choisis et assis dans son fauteuil à l'officier non loin de "La mort du général Wolfe", je me suis constitué prisonnier pour mieux l'approcher et enfin le tuer." $(P E, 125)$ Le narrateur amorce un processus d'identification qui l'amène à se constituer prisonnier. Quelques minutes plus tard, l'identification atteint une telle perfection qu'elle se retourne contre lui et le place non plus dans la position de l'assassin mais de l'assassiné : "Il me semble que je ne pourrai jamais plus sortir d'ici. Et tandis que l'après-midi lumineux oblique vers la Barre des Écrins, je suis enfermé avec mon mobilier funéraire." (PE, 130) Dans le château, il perd peu à peu la supériorité qu'il croyait avoir, jusqu'à se sentir "sur le point de céder à la fatigue historique": "Les coordonnées de l'intrigue se sont entremêlées. J'ai perdu le fil de mon histoire, et me voici dans un chapitre que je ne sais plus comment finir." $(P E, 136) \mathrm{La}$ métanarration resurgit alors dans la narration actancielle pour avouer sa désespérance face à cette intrigue qui s'échappe comme par la force d'attraction d'une narration étrangère et antagoniste.

Le coup fatal est porté lorsque $\mathrm{H}$. de Heutz pénètre dans le château et se précipite pour répondre au téléphone: le contre-révolutionnaire

19. Les jeux de double et de masque ont déjà été largement commentés par la critique; nous ne reprenons ici que les traits essentiels à notre argumentation. Voir, entre autres, Patricia Smart, Hubert Aquin, agent double. La dialectique de l'art et du pays dans Prochain épisode et Trou de mémoire, Montréal, Presses de l'Université de Montréal, 1973; René Lapierre, Les masques du récit. Lecture de Prochain épisode de Hubert Aquin, Montréal, Hurtubise/HMH, 1980. 
échange alors quelques paroles avec une femme qui, selon les indices disséminés au long du roman, semble bien être $\mathrm{K}$, la femme désirée par le révolutionnaire. Plus encore, $\mathrm{H}$. de Heutz accepte le rendez-vous qu'elle lui fixe à l'Hôtel d'Angleterre à six heures trente, lieu et heure où le narrateur devait lui-même la rencontrer. Il ne peut être ici question d'un délire paranoïaque du narrateur, mais d'une structure narrative fondée sur la répétition. Cela permet au contre-révolutionnaire d'intégrer à l'histoire qu'il représente chaque geste du révolutionnaire, jusqu'à confondre les flammes cubaines avec les eaux calmes' du lac Léman. Ce que met en scène Aquin, outre une certaine situation coloniale, c'est en quelque sorte "la ruse de la raison" de $\mathrm{Hegel}^{20}$ : les désirs et les passions les plus hétérogènes, et parmi elles les volontés révolutionnaires, sont présentés comme s'ils participaient tous d'une histoire transcendante qui les utilise comme simples instruments au service d'une fin qui les dépasse. Chez Aquin, cette conception de l'histoire (où les buts individuels ne sont que les moyens au service d'un déploiement transcendant) se métamorphose en une lutte des narrativités à la suite de laquelle la révolution se trouve constamment réinscrite, non plus par une ruse de la raison, mais par une ruse de la narrativité, dans la trame historique qu'elle tentait de rompre. Par la répétition qu'il associe à cette ruse narrative utilisée par $\mathrm{H}$. de Heutz, le romancier met en lumière l'élément régressif d'une conception de l'histoire articulée sur un temps continu et linéaire, dont l'itinéraire serait en germe dans l'Origine, et qui avancerait vers sa propre finalité. L'histoire, selon cette conception, n'est plus qu'un réservoir d'événements qui se répètent sans cesse, depuis un passé déjà connu et une Origine transcendante, vers un avenir entièrement tracé d'avance, jusqu'à une Fin inexorable.

\section{La répétition de "L'art de la défaite "}

Le spectre de la répétition hante donc la totalité du roman d'Aquin sous diverses formes: le ressouvenir des révolutions passées dans l'autonarration, la reprise des matrices romanesques et l'assujettissement au poids de l'histoire dans la métanarration, la répétition pure du discours du narrateur par $\mathrm{H}$. de Heutz, et l'identification du révolutionnaire au contre-révolutionnaire dans la narration actancielle. Il faudrait ajouter un dernier phénomène de répétition, extra-romanesque celui-là. Comme l'a

20. “Cette masse immense de désirs, d'intérêts et d'activités constitue les instruments et les moyens dont se sert l'Esprit du Monde pour parvenir à sa fin, l'élever à la conscience et la réaliser. Car son seul but est de se trouver, de venir à soi, de se contempler dans la réalité. C'est leur bien propre que peuples et individus cherchent et obtiennent dans leur agissante vitalité, mais en même temps ils sont les moyens et les instruments d'une chose plus élevée, plus vaste qu'ils ignorent et accomplissent inconsciemment." G. W. F. Hegel, La raison dans l'bistoire, trad. K. Papaioannou, Paris, Union générale d'éditions, coll. ‘ 10/18", 1965, p. 110. 
déjà remarqué Anthony Purdy ${ }^{21}$, l'échec apparent de la tentative révolutionnaire reprend la structure narrative d'une autre insurrection qu'Aquin avait analysée dans un essai paru la même année que Prochain épisode. Dans "L'art de la défaite", il interprétait les rébellions de 1837-1838 et une répression violente, comme une "véritable anthologie d'erreurs sanglantes, de négligences et d'actes manqués ${ }^{22}$. Considérant non seulement l'historiographie comme une narration, mais aussi le processus historique comme un récit en marche, il décrit l'incapacité des Patriotes canadiens à profiter de leur avantage sur les troupes anglaises après une première victoire à Saint-Denis :

On se croirait à la représentation d'une tragédie classique, à l'instant où le chœur, instantanément et dans une invraisemblable simultanéité a un blanc de mémoire: c'est un silence de mort. [...] Le chœur ne peut pas continuer parce que les autres acteurs n'ont pas dit les paroles qu'ils devaient dire; cette hypothèse nous permet de comprendre ce qui se passe sur la scène. Le chœur, figé de stupeur, ne peut pas enchaîner si l'action dramatique qui vient de se dérouler n'était pas dans le texte; les Patriotes n'ont pas eu un blanc de mémoire à Saint-Denis, mais ils étaient bouleversés par un événement qui n'était pas dans leur texte : leur victoire ${ }^{23}$ !

Ainsi, l'échec de la rébellion s'expliquerait d'abord par un trou de mémoire, par une perte du fil de la narration, puis, plus profondément, par l'inadéquation de l'entreprise des insurgés au grand livre de l'histoire. Ici, tout comme dans le roman policier mis en abyme dans Prochain épisode, les acteurs de l'histoire, les révolutionnaires, ont à affronter une narration qui les précède, qui les intègre invariablement à elle et leur interdit d'accéder à l'histoire.

Plus encore, Aquin propose comme seconde explication à la défaite la façon tout anglaise qu'avaient les Patriotes de livrer bataille: "On mange comme son hôte. On se bat comme lui: on fait la guerre aux Anglais exactement comme il nous ont appris à faire la guerre, sous leurs ordres, aux Américains en $1812^{24}$. "La structure narrative d'identification à l'ennemi qui pointe dans le roman policier refait ici surface: les protagonistes se reconnaissent dans la défaite qu'ils souhaitent à leurs ennemis, jusqu'à s'approprier leur échec. De ce point de vue, Prochain épisode, qui regorge de références à cet événement fondateur de l'histoire québécoise, serait la répétition d'une forme narrative centrée sur l'échec et sur l'impossibilité pour le peuple québécois de passer à l'histoire. Les répétitions à l'intérieur du roman reprennent ainsi, comme un archétype aveuglant,

21. Anthony Purdy, "De "L'art de la défaite" à Prochain épisode: un récit unique?», Voix et Images, vol. $\mathrm{X}, \mathrm{n}^{\circ} 3$, printemps 1985, p. 113-125. Cet article nous semble fondamental pour toute lecture de Prochain épisode.

22. Hubert Aquin, "L'art de la défaite: considérations stylistiques", Mélanges littéraires II, op. cit., p. 131.

23. Ibid., p. 134

24. Ibid., p. 136 . 
l'échec des Patriotes qui lui-même semble fonder une certaine mémoire collective :

Ce qui m'afflige encore plus c'est que leur aventure ratée avec insistance véhicule, de génération en génération, l'image du héros vaincu: certains peuples vénèrent un soldat inconnu, nous, nous n'avons pas le choix: c'est un soldat défait et célèbre que nous vénérons, un combattant dont la tristesse incroyable continue d'opérer en nous, comme une force d'inertie ${ }^{25}$.

L'échec suprême est donc la répétition - par sédimentation de la tradition - d'un échec originel posé comme naissance de l'histoire, d'un commencement paradoxal symbolisant l'être de la collectivité par la négation de son devenir. C'est peut-être ici qu'apparaît l'appartenance profonde du roman d'Aquin à ce que Deleuze et Guattari nommaient les littératures mineures ${ }^{26}$ : l'impossibilité à la fois d'écrire et de ne pas écrire se module dans Prochain épisode en incapacité de l'écrivain à réussir une ouvre québécoise (en vertu de cet art de la défaite), et l'impossibilité de ne pas écrire cette œuvre vouée à l'échec. Au plan collectif, il y a à la fois impuissance à passer à l'histoire et à se passer de l'histoire. Tout ce réseau complexe de doubles contraintes contamine le roman jusqu'à lui donner la forme de sa propre abolition, celle d'un roman dont le titre marque en quelque sorte la négation. Le roman est à l'image de ces messages passés de main en main par les conspirateurs et que le dernier destinataire doit brûler après avoir pris connaissance des instructions devant mener à l'insurrection ultime.

\section{Le simulacre: roman-événement, répétition parodique, abolition de l'archétype}

On pourrait croire que le roman d'Aquin affirme l'impossibilité de toute révolution digne de ce nom, qu'il exhibe la répétition aliénante à l'œuvre dans l'histoire et qu'il annonce, malgré l'appel à un prochain épisode, l'échec sans cesse repris des révolutions passées et à venir. Ce constat voilerait cependant la duplicité sémantique du terme "révolution" que sous-entend Aquin dans un article sur la répétition chez James Joyce, "notre seul et abominable professeur en déséquilibre, notre guide unique et complètement désaxé ${ }^{27}$. "Dans un texte aussi bref que pénétrant,

25. Ibid., p. 137.

26. Sur l'impasse rendant la littérature “impossible " pour Kafka et les juifs de Prague, voir Gilles Deleuze et Félix Guattari, Kafka. Pour une littérature mineure, Paris, Minuit, coll. "Critique *, 1975, p. 29-30. L'essai capital d'Aquin sur le statut de l'écrivain québécois comme "non-écrivain absolu " s'inscrit dans une problématique analogue (Hubert Aquin, "Profession écrivain ", Point de fuite (1971), Montréal, Bibliothèque québécoise, 1995, p. 45-59).

27. Hubert Aquin, "Littérature et aliénation", Mélanges littéraires II: Comprendre dangereusement, p. 253. D'après les responsables de l'édition critique, la lecture d' Ulysse de Joyce par Aquin remonterait au moins à 1952, soit plus de dix ans avant la rédaction de Prochain épisode, époque à laquelle il projetait l'écriture d'une thèse intitulée Phénoménologie de la création du personnage dans le roman et qui portait, entre autres, sur l'auteur irlandais. 
intitulé "De Vico à James Joyce, assassin d'Ulysse", Aquin avance trois thèses fondamentales pour la compréhension de la relation dialectique entre l'histoire et la narrativité romanesque. Premièrement, le roman de Joyce serait essentiellement un roman historique, non parce qu'il intègre à sa narration un nombre incalculable de références à l'histoire, mais parce qu'il se pose lui-même comme un événement n'expliquant rien mais devant au contraire être interprété : "Le livre de Joyce se substitue à l'événement qu'un historien cherche à circonscrire et à comprendre. Ulysse de James Joyce est son propre événement; il fait désormais partie de l'histoire en tant qu'événement qu'on peut mesurer, étalonner, comprendre et ranger avec la méthode de l'historien ${ }^{28}$." Deuxièmement, l'originalité propre à Joyce résiderait dans son absence d'originalité, c'est-à-dire sa répétition de L'odyssée d'Homère:

L'originalité de ce livre n'est pas d'être original. Son thème répète ("history repeating itself with a difference ") L'odyssêe d'Homère qui, à l'origine, était autant un livre d'histoire qu'une épopée. Joyce répète Homère, l'aède aveugle, qui lui-même a répété les récits des exploits d'Ulysse racontés par d'autres aèdes. On sait à quel point Joyce chérissait la philosophie de l'histoire de Vico. En fait, il a composé sa propre épopée (répétition parodique de L'odyssée) selon un schéma typiquement vicéen ${ }^{29}$.

Troisièmement, la répétition qu'effectue Joyce à partir du mythe homérique serait non pas l'expression d'une matrice antérieure régissant son œuvre comme un archétype, mais la mise à mort d'une transcendance ayant dirigé comme une main de fer l'histoire du roman:

Joyce était obsédé, semble-t-il par la redondance de l'histoire, séduit aussi par cette répétition toujours recommencée et fier d'ajouter sa touche homérique aux variantes de Virgile et de Dante. [...] Dans son cas, la différence propre de la répétition est énorme; la répétition cyclique atteint en lui un point culminant. Bloom est la fin des voyages d'Ulysse! Joyce "historien " a voulu tuer Ulysse en le réincarnant dans Léopold Bloom et il a réussi. Cette ambition du grand romancier irlandais n'a jamais été signalée, ni avouée à plus forte raison; mais elle correspond bien à l'orgueil démesuré et nietzschéen de Joyce. Comme Dieu, Ulysse est mort ${ }^{30}$.

Les trois thèses conviennent parfaitement à Prochain épisode: roman empreint d'histoire, mais qui constitue par sa modernité radicale non une explication historique mais un pur événement toujours à interpréter; roman qui ne cesse de reprendre des schèmes narratifs antérieurs et constitue son originalité par le refus du "Graal esthétique"; roman qui répète une première narration, celle des rébellions de 1837-1838, non pour la confirmer mais pour mettre à nu sa valeur archétypale avant son

28. Hubert Aquin, "De Vico à James Joyce, assassin d'Ulysse" (1973), Mélanges littéraires II: Comprendre dangereusement, p. 327.

29. Ibid., p. 328.

30. Ibid. 
abolition. Roman-événement, répétition parodique et abolition de l'archétype: sous ces trois traits, qu'Aquin décèle dans l'œuvre de Joyce et qui s'appliquent sans reste à Prochain épisode, se dessine une logique paradoxale ressemblant étrangement à la théorie du simulacre que Gilles Deleuze conceptualise à partir du platonisme et qu'il oriente lui aussi dans une perspective nietzschéenne et anti-hégélienne ${ }^{31}$. Dans La république, Platon exposait sa théorie des Idées par la métaphore des trois lits: il y aurait un premier lit, créé par le démiurge, ensuite un second, fabriqué par l'artisan, puis un troisième, rendu par le poète et le peintre. Le lit du démiurge serait une pure Idée, le lit de l'artisan une copie de cette Idée, et le lit de l'artiste un simulacre, une copie de la copie, impure et condamnable. Selon l'interprétation de Deleuze, l'artisan reproduirait donc - répéterait - le lit idéel que le démiurge a créé, mais le lit de l'artiste reproduirait non seulement le lit de l'artisan, mais plus essentiellement la répétition première: c'est-à-dire que le simulacre créé par l'artiste se donnerait comme la copie d'une copie et reproduirait non l'identité entre le lit idéel et le lit artisanal, mais leur différence irréductible - le simulacre produirait ainsi la différence, en répétant la distance entre le fondement et la copie, en abolissant la transcendance de l'archétype.

L'argument d'Aquin relève d'une logique étrangement analogue: Joyce réitérerait avec Ulysse une première répétition, celle que le paradigme homérique a imposé à l'histoire du roman; mais ce faisant, il en détruirait le fondement et s'en affranchirait ultimement pour affirmer sa différence irréductible, une pure événementialité. La répétition joycienne ne s'attacherait donc pas à l'origine, mais la remettrait en jeu dans la différence et arriverait ainsi à rompre avec elle par une pratique foncièrement parodique. De la même façon, on peut lire dans Prochain épisode plusieurs répétitions préexistantes, celles de l'histoire, de sa narration, de sa métanarration qui reprend sa propre situation d'enfermement. Ces répétitions, Aquin ne les confirme pas dans leur valeur fondatrice ou originaire; il les traduit en purs simulacres afin de s'en délester. Tout autrement, la répétition archétypale ou monocentrée dans Prochain épisode serait celle au service de laquelle œuvre $\mathrm{H}$. de Heutz: celle d'une histoire qui se constitue peu à peu en révélant ses fondements immuables et son immobilisme foncier par des ruses diverses, celle des grandes matrices narratives et historiques qu'affronte avec peine le métanarrateur, et encore celle du ressouvenir où le narrateur ne cessait de se perdre dans les images du passé. La répétition de la répétition, nomade et excentrée, qui annihile le fondement, qui évacue la reproduction du Même, qui est au contraire un retour de l'altérité, serait produite par Prochain épisode en entier tel qu'il

31. Pour une explication succincte de cette théorie, voir Gilles Deleuze, "Platon et le simulacre", Logique du sens, Paris, Minuit, coll. "Critique", 1969, p. 292-107; consulter aussi Gilles Deleuze, Différence et répétition, Paris, Presses universitaires de France, 1968. 
est conçu par Aquin: une répétition qui en dévoilant ses fondements les déconstruit aveuglément, violemment, comme Joyce a pu le faire avec L'odyssée. De même qu'Ulysse meurt sous les coups de marteau de Joyce, "l'art de la défaite" doit connaître son terme avec Prochain épisode.

Le romancier suggère d'ailleurs cette lecture par une mise en abyme éclairante: son narrateur, désespéré entre les mains de l'ennemi, se dit contraint de prendre son "masque de grand déprimé ", c'est-à-dire de feindre l'échec pour s'assurer la victoire. De même que "condamné à une langue étrangère, [Joyce] s'est mystérieusement vengé en la rendant étrangère à elle-même ${ }^{32}$ ", Aquin, lui-même condamné à une histoire étrangère, s'est vengé en la travestissant jusqu'au simulacre. Feindre l'échec pour s'assurer la victoire, c'est tout le mouvement de Prochain épisode.

\section{La lettre et l'esprit de la révolution}

Les propos de Marx sur la répétition de l'histoire et particulièrement lors des époques révolutionnaires sont trop étroitement liés au propos de Prochain épisode pour qu'on omette de les rappeler. "Hegel fait quelque part cette remarque que tous les grands événements et personnages de l'histoire mondiale surgissent pour ainsi dire deux fois. Il a oublié d'ajouter: la première fois comme tragédie, la seconde fois comme farce ${ }^{33}$." Marx distingue deux types d'événementialité à l'œuvre dans l'histoire: une première, essentiellement tragique et qui reprend l'esprit révolutionnaire dans ce qu'il a de plus digne, puis une seconde, foncièrement grotesque, où la lettre révolutionnaire est répétée aveuglément avec tout ce qu'elle possède d'inessentiel. Encore prisonnier du modèle platonicien qu'il renversera en partie, tout en conservant la structure oppositionnelle des mondes sensible et intelligible, Marx valorise la répétition comme produit d'une essence objective (la nécessité historique et matérielle) et la rejette comme simulacre et reprise théâtrale (l'imaginaire idéologique). Respectant la tripartition du modèle de Platon (Idée, copie, simulacre), il reprend donc mot à mot la condamnation, contenue dans La république, de la répétition de la répétition. Nuançant son propos, Marx reconnaît néanmoins la force que celle-ci peut offrir à la praxis dans une révolution de type tragique, où coïncident idéologie et réalité matérielle :

La résurrection des morts, dans ces révolutions, servit par conséquent à magnifier les nouvelles luttes, non à parodier les anciennes, à exagérer dans l'imagination la tâche à accomplir, non à fuir sa solution dans la réalité, à retrouver l'esprit de la révolution et non à faire revenir son spectre ${ }^{34}$.

32. Hubert Aquin, "La fatigue culturelle du Canada français ", Mélanges littéraires II, op. cit., p. 100.

33. Karl Marx, Le dix-huit brumaire de Louis Bonaparte, trad. G. Cornillet, Paris, Éditions sociales, coll. "Essentiel „, 1992 [1852], p. 69.

34. Ibid., p. 71. 
Approfondissant encore ce recours au passé - ce qui ne peut que paraître étonnant au sein d'une philosophie téléologique - , Marx use d'une métaphore linguistique d'une importance fondamentale pour la lecture de Prochain épisode:

C'est ainsi que le débutant qui a appris une nouvelle langue la retraduit toujours dans sa langue maternelle, mais il ne se sera approprié l'esprit de cette nouvelle langue et ne sera en mesure de s'en servir pour créer librement que quand il saura se mouvoir dans celle-ci sans réminiscence, en oubliant en elle sa langue d'origine ${ }^{35}$.

La répétition tragique dans l'histoire se compare donc à l'apprentissage d'une langue seconde dont on ne réussirait d'abord à percer la logique propre, la sémantique unique, qu'en la traduisant compulsivement dans sa langue maternelle. Ainsi, en termes politiques, à la volonté révolutionnaire se juxtaposerait dans la conscience idéologique un retour à l'origine, au mythe de fondation de la société, retour qui permettrait précisément de penser un nouveau commencement. Pour filer la métaphore marxienne, le roman d'Aquin pourrait se trouver à une telle frontière entre deux langues ou, plus précisément, entre deux discours sur l'histoire: un premier qui chercherait à comprendre une nouvelle situation historique dans les termes des époques antérieures, selon une logique de l'Origine qui le voue à la répétition du passé comme archétype du présent; un second qui se présenterait sous un jour entièrement nouveau et qui serait appelé par le titre du roman comme un "prochain épisode", mais qui ne serait accessible que par la répétition du discours antérieur et, d'une certaine façon, par sa reconnaissance critique. En fait, Prochain épisode s'efforce de se maintenir exactement à ce point entre l'abolition d'un discours ancien et l'épiphanie d'un discours inédit.

Cette ouverture à une prochaine énonciation, paradoxalement appelée par la répétition d'un énoncé précédent dont le sens se trouve ainsi modifié, constitue la pragmatique narrative profonde du roman. Il est d'ailleurs possible qu'une des seules possibilités de représenter la révolution dans un récit soit non de la dire mais de la montrer, c'està-dire de l'inscrire dans la structure même du langage plutôt que dans son contenu, sous une forme essentiellement performative où l'énoncé contiendrait sa propre abolition. Le récit aquinien, avec son fort coefficient de répétition, tente une telle entreprise en subvertissant non l'objet du récit mais sa pragmatique même. L'on pourrait dire, pour paraphraser Lyotard ${ }^{36}$, que la révolution occupe l'espace du différend entre deux phrases qui ne sont pas seulement de régimes différents, mais qui s'inscrivent dans des genres discursifs hétérogènes. En effet, la révolution n'est jamais "phrasable", puisqu'elle se tient entière dans le passage d'une

35. Ibid., p. 70.

36. Jean-François Lyotard, Le différend, Paris, Minuit, coll. "Critique", 1983. 
phrase à l'autre, ou, comme le dirait Marx, d'une langue à une autre. Ainsi, la révolution n'appartiendrait jamais au dit; elle n'aurait pour seul recours que le dire. Par le titre même de son roman, Aquin réussit là où son narrateur avait échoué. En écrivant un roman qui se présente comme l'annonce d'un épisode tout autre, il montre la révolution à l'œuvre, qui abolit l'histoire (et, en quelque sorte, le roman) et restaure - du moins dans l'économie d'un récit fictif - l'expérience du présent historique et de son potentiel immense de bifurcations, dégagée d'une histoire ployant sous le poids de son passé.

Porter la négativité de la dialectique historique jusqu'à sa négation, c'est la stratégie retorse du roman d'Aquin: faire triompher l'histoire pour mieux dévoiler sa face cachée, c'est-à-dire la nature profondément régressive, conservatrice, tant d'une idéologie du progrès que du mythe d'une origine fondatrice, puis par le titre nier l'œuvre entière ainsi que la conception de l'histoire qui en ressortait victorieuse. "Chose certaine, mon projet tient du défi en cela même qu'il peut sembler, selon la logique courante de notre métier, l'initiative contre-indiquée par excellence. Dans cette allure illogique réside toutefois sa qualité redoutable: c'est le contredéguisement!" $(P E, 111)$ Singulière représentation du temps de l'histoire: les catégories narratives du début et de la fin lâchant prise sur le roman, on assiste à l'apparition d'une temporalité étrangère face au fil judéochrétien (avec sa genèse transcendante et sa fin immanente), à l'image d'un moteur qui semble tourner seul, sans opérateur au départ, sans production à l'arrivée autre que son fonctionnement propre. Prochain épisode est la diabolique mise en pièces de la conception traditionnelle de l'histoire comme mouvement linéaire et unidirectionnel orientée depuis une origine vers son terme. Gilles Marcotte remarquait à propos du roman:

Voilà bien cependant une étrange forme de conscience historique, qui abolit le passé et non seulement fait de l'événement " un acte sans racines temporelles, un pur surgissement, mais encore le prive de tout développement. Car l'événement, selon le narrateur de Procbain épisode, sera "absolu et répété "; il n'engendrera pas une continuité historique, mais il s'insère d'avance dans la structure mythique de l'éternel recommencement ${ }^{37}$.

Prochain épisode participe sans doute de cette littérature québécoise de l'inachèvement qui marque les années soixante, mais en aucun cas il ne refuse au présent son passé - la surcharge de références historiques suffirait à démontrer le contraire - ni ne s'enferme dans une histoire réglée sur le modèle de l'éternel retour. Au contraire, on remarquera que la répétition remplit la double fonction de reconnaître le passé, de le rappeler à la mémoire, puis de restituer au présent sa contingence et sa différence irréductible. Car il n'y a pas lieu ici d'évoquer un refus de l'histoire, puisque la répétition a d'abord pour finalité l'assomption du

37. Gilles Marcotte, op. cit., p. 238. 
devenir nécessaire à toute expérience du présent de l'histoire, c'est-à-dire de sa charge événementielle. La négativité si souvent reconnue dans Prochain épisode n'a donc pas pour objet les illusions révolutionnaires, mais bien les utopies jumelles de la téléologie historique et de l'histoire providentielle qui ne lèguent au présent historique que les traits d'un musée sans vie où tout est déjà joué. Et, répétons-le, cette négativité ne récuse pas l'historicité, mais engage une réflexion sur les formes de l'histoire se résumant finalement par ces deux traits: "la lutte contre l'illusion de fatalité créée par la rétrospection historique et le plaidoyer pour la contingence du présent requise par l'action politique ${ }^{38}$." Sceptique à l'égard de possibles effets pervers de la mémoire collective et de l'historiographie, Aquin rappelle que l'histoire n'est ni ce qui est déjà advenu ni une continuité sans faille, mais aussi un temps collectif qui se conjugue au présent, un temps porteur de possibilités sans commune mesure avec une quelconque causalité historique. Le modèle révolutionnaire qui imprègne Prochain épisode ne dit pas autre chose: la seule possibilité de faire l'histoire est d'assumer sa triple temporalité tout en revendiquant la part de contingence des temps actuels. Et cette leçon de choses, tant dans son argumentation que dans ses conséquences, on le voit, dépasse largement le cadre des préoccupations d'une paradoxale "Révolution tranquille".

38. Nous reprenons ici les propos de Ricœur où il définit, s'inspirant de l'Introduction à la philosophie de l'bistoire de Raymond Aron, deux des foyers majeurs de l'historiographie. Il cite aussi cette phrase d'Aron qui pourrait s'appliquer au projet romanesque aquinien: "L'enquête causale de l'historien a moins pour sens de dessiner les grands traits de relief historique que de conserver ou de restituer au passé l'incertitude de l'avenir.. Paul Ricœur, Temps et récit. 1. L'intrigue et le récit bistorique, Paris, Seuil, coll. "Points *, 1991 [1983], p. 331-332. 